\title{
La diabetes mellitus
}

\section{Herramienta para la toma de decisiones compartidas en la diabetes mellitus tipo II}

\author{
Leticia Gimeno PitARCH \\ al337848@uji.es \\ Rosa Álvaro Ballester \\ al318269@uji.es \\ GLORIA DE LA TORRE MONFORT \\ al319535@uji.es \\ Mariana Claudia Matel \\ al317225@uji.es \\ LUIS VICENTE LIZÁN TUDELA \\ lizan@med.uji.es
}

\section{Resumen}

La Diabetes es una enfermedad crónica y prevalente, que tiene un desarrollo gradual y para cuyo control son esenciales el consejo y la guía del personal sanitario, junto con la atención del paciente. Por lo tanto, resulta imprescindible que el paciente conozca y entienda su patología junto con las opciones para su tratamiento, cómo afecta a su calidad de vida, así como que pueda valorar sus propias preferencias y sepa dónde ha de buscar información. Se ha elaborado una herramienta de ayuda con el objetivo de proporcionar información sobre las diferentes opciones de tratamiento, de manera que facilite la reflexión y la discusión conjunta con el profesional. El resultado ha sido la creación de un tríptico. En conclusión, se ha conseguido implementar un instrumento de ayuda para que los pacientes conozcan más detalladamente acerca de su patología y puedan tomar decisiones compartidas con el profesional sanitario según sus preferencias, aumentando la confianza en el sistema de salud y la adherencia al tratamiento.

Palabras claves: diabetes, tratamiento, preferencias, información, herramienta de ayuda.

\section{Abstract}

Diabetes is a chronic and prevalent disease, which has a gradual development and for its control are essential advice and guidance of health personal, together with the patient's attention. Therefore, it is imperative that the patient knows and understands 
their pathology along with the options for their treatment, how it affects their quality of life, as well as being able to value their own preferences and look for where to look for information. An aid tool has been developed with the objective of providing information on the different treatment options, so as to facilitate reflection and joint discussion with the professional. The result has been the creation of a triptych. In conclusion, an instrument has been implemented to help patients to know more about their pathology and to make decisions that are shared with the healthcare professional according to their preferences, increasing confidence in the health system and adherence to treatment.

Keywords: diabetes, treatment, preferences, information, help tool.

\section{Introducción}

La prevalencia de la diabetes tipo 2 se ha convertido en uno de los problemas sanitarios más graves de nuestro tiempo. Según un estudio llevado a cabo por el Centro de Investigación Biomédica en Red de Diabetes y Enfermedades Metabólicas Asociadas, y la Sociedad Española de Diabetes, existen 246 millones de personas afectadas en todo el mundo, una cifra que sigue amentando y que va a suponer uno de los principales motivos de consulta en atención primaria.

La Diabetes Mellitus aparece cuando el páncreas no produce suficiente insulina o cuando el organismo no utiliza eficazmente la que produce. Se trata de una enfermedad crónica, con la que el paciente tendrá que aprender a convivir, adaptando su estilo de vida a las nuevas necesidades.

Cabe mencionar, que el buen control de la diabetes es clave, no solo para asegurar la calidad de vida de los pacientes según afirma Pablos-Velasco, Pedro, Emilio Salguero-Chaves, Julio Mata-Poyo, Beatriz Derivas-Otero, Ricardo García-Sánchez y Pablo Viguera-Ester en su artículo Quality of Life and Satisfaction with Treatment in Subjects with Type 2 Diabetes (2014), sino también para evitar posibles complicaciones como la afectación microvascular (retinopatía diabética, nefropatía diabética, neuropatía periférica...), o bien, la macrovascular; ya que la Diabetes Mellitus está considerada como una enfermedad asociada al riesgo cardiovascular, siendo ésta la primera causa de muerte entre los diabéticos. Tal y como se determinó en un estudio realizado sobre Estadísticas y Causas de Mortalidad por parte de M. Salgado Pineda, J. Franch Nadal, M. Pallas Ellacuria, C. Oriol Zerbe, J. Grau Bartomeu y J. Castellà García (2001), «La principal causa de muerte en diabéticos tipo 2 fue la enfermedad cardiovascular $(47,7 \%)$, mientras que en el Registro Civil la mortalidad por esta razón es claramente menor (...). Esto sugiere que debemos insistir aún más en la importancia de monitorear los factores de riesgo cardiovascular.»

Todo ello ha conllevado un gran impulso en el desarrollo de nuevas terapias antidiabéticas, lo que puede causar cierta incertidumbre a la hora de prescribir el mejor tratamiento, ya que cada uno de ellos presenta una serie de ventajas y desventajas que tanto paciente como médico deberán valorar. Como estudiantes de $3^{\circ}$ Medicina hemos sido testigos, durante nuestras prácticas externas, de la gran cantidad de pacientes con Diabetes Mellitus tipo II mal controlada y por lo tanto, con necesidad de modificar su tratamiento.

Definido el origen del estudio, nuestro objetivo principal ha sido elaborar una herramienta de autoayuda dirigida a los pacientes con Diabetes Mellitus tipo II, con necesidad de modificar y adaptar el tratamiento, teniendo en cuenta sus preferencias. 
Según Bosch J.M., (2012), en La toma de decisiones conjuntas en medicina, «La participación del paciente en la toma de decisiones (PPTD) precisa un cambio en la forma tradicional de la atención sanitaria, pasando de un modelo paternalista a una relación más colaborativa, donde la opinión del paciente (y sus familiares) comporta ceder parte del control de la consulta, un trabajo de negociación continuado, y si nos atenemos a respetar las decisiones del paciente, un claro ejercicio que le permita ejercer su autonomía, lo que constituye un imperativo ético». Además, según Ruiz Moral R., Peralta Munguia L., Pérula de Torres LA., Olloqui Mundet J, Carrión de la Fuente T, Sobrino López A y cols. (2012), la mayoría de los pacientes tras una consulta concreta desean poder dar su opinión a las propuestas de tratamiento que surgen, sin embargo perciben que sus médicos raramente les ofrecen estas oportunidades de participación. Por lo tanto, partiendo de la evidencia encontrada, la posibilidad de crear un instrumento que logre y asegure la toma de decisiones compartidas médico-paciente, supone un gran objetivo a conseguir.

\section{Método}

Según The BMJ (2013), una plataforma involucrada en el debate sobre la salud, cada vez más pacientes manifiestan su deseo de participar activamente en las decisiones relacionadas con su tratamiento. Siendo la cifra cada vez mayor, aparecen nuevas necesidades a la hora de afrontar una decisión compartida médico-paciente, por lo que el objetivo de nuestro estudio se basa en proporcionar la información necesaria a los pacientes sobre las diferentes opciones de tratamiento de manera que facilite la reflexión y discusión con el profesional sanitario a la hora de tomar decisiones compartidas.

El método utilizado ha sido la creación de una herramienta de autoayuda, que es un instrumento para facilitar la toma de decisiones compartidas sobre el tratamiento de la Diabetes Mellitus, lo cual se consigue con la aportación de información a los pacientes sobre su enfermedad. Según Coulter A., Parsons S. y Askham J (2008), la alfabetización en salud es fundamental para mejorar la participación de los pacientes en su atención, y por lo tanto todas las estrategias para fortalecer la participación de los pacientes deben tener como objetivo mejorarla.

Esta herramienta ha de ser clara, concisa, sencilla, visual, breve, divulgativa y sobre todo accesible para aquellos a los que estaba dirigida, es decir, pacientes con Diabetes Mellitus Tipo 2 mal controlada, que acuden al centro de atención con el objetivo de modificar y mejorar su tratamiento. De la misma forma, tal y como afirman Jiménez De Gracia L., Ruiz Moral R., Gavilán Moral E., Hueso Montoro C., Cano Caballero Gálvez D., y Alba Dios MA (2012), ha de ser un instrumento práctico que pueda incorporarse fácilmente en la consulta, y en la rutina del día a día de los médicos, en este caso, de atención primaria.

\section{Resultados}

El resultado obtenido ha sido la elaboración de un tríptico que contiene una portada en la que se presenta la diabetes como patología de forma breve, clara y sencilla. En su interior, se define la enfermedad junto a los niveles de glucosa plasmática en ayunas que la determinan. Al mismo tiempo, se especifican los diferentes tipos de diabetes y cómo puede llegar esta a repercutir en la calidad de vida de los pacientes, con el objetivo de que sean conocedores de los posibles síntomas que se pueden presentar a lo largo del tiempo. 
La información encontrada en el tríptico se extrae de una serie de asociaciones internacionales sobre la diabetes como es el caso de American Association of Clinical Endocrinologists (AACE), American Diabetes Association (ADA), Canadian Diabetes Association (CDA).

Además, se ha incluido un apartado de opciones terapeuticas para proporcionar información al paciente acerca de los distintos tratamientos disponibles. El apartado más característico de la herramienta, ha sido el de preferencias del paciente, en el cual, se han incluido una serie de cuestiones para que este valore los diferentes tratamientos de acuerdo a sus características, necesidades e intereses y pueda tomar una decisión conjunta con su médico. Al mismo tiempo se ha incluido una check list basada en los criterios de la International Patient Decision Aid. Standards. Por último, se ha considerado de gran importancia proporcionar al paciente páginas web dónde pueda acceder y ampliar sus conocimientos acerca de la patología y les pueda servir como guía.

El conocimiento sobre la farmacología de la diabetes se basa en el Manual de Farmacología y terapéutica, Brunton, L., Parker, K., Blumenthal, D. y Buxton, I. (2009). Goodman y Gilman. Sección XII, Hormonas y sus antagonistas, insulina, hipoglucemiantes orales y la farmacología del páncreas endocrino (páginas 1037-1059). Mc Graw Hill, y en el libro Farmacología Humana ( $5^{a}$ Edicicón), J.C. Flórez, K. Freijanes y J. Flórez. Insulina y Hormonas antidiabéticas (pàgines 1051-1073).

A continuación se incluye el resultado de este trabajo:
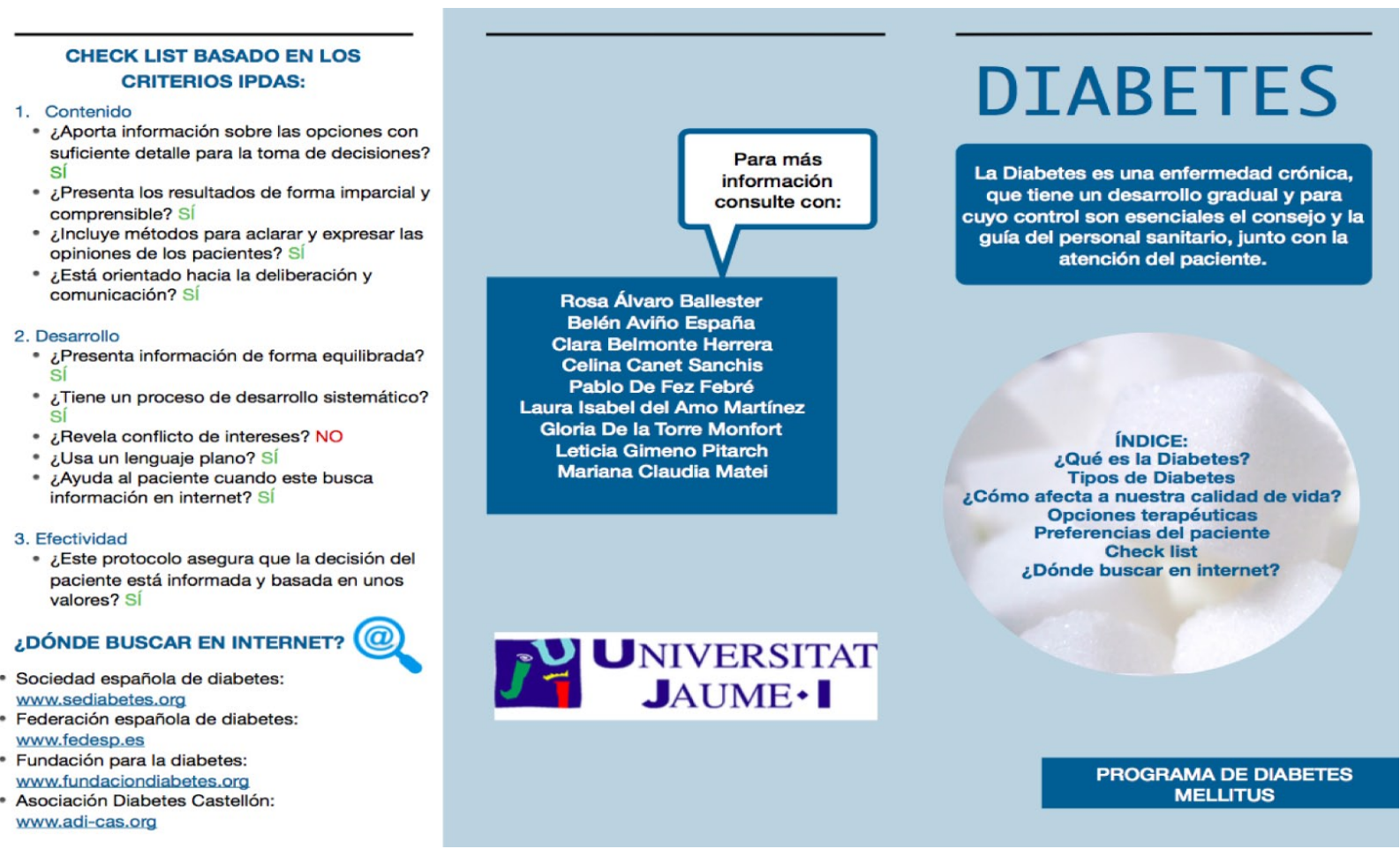

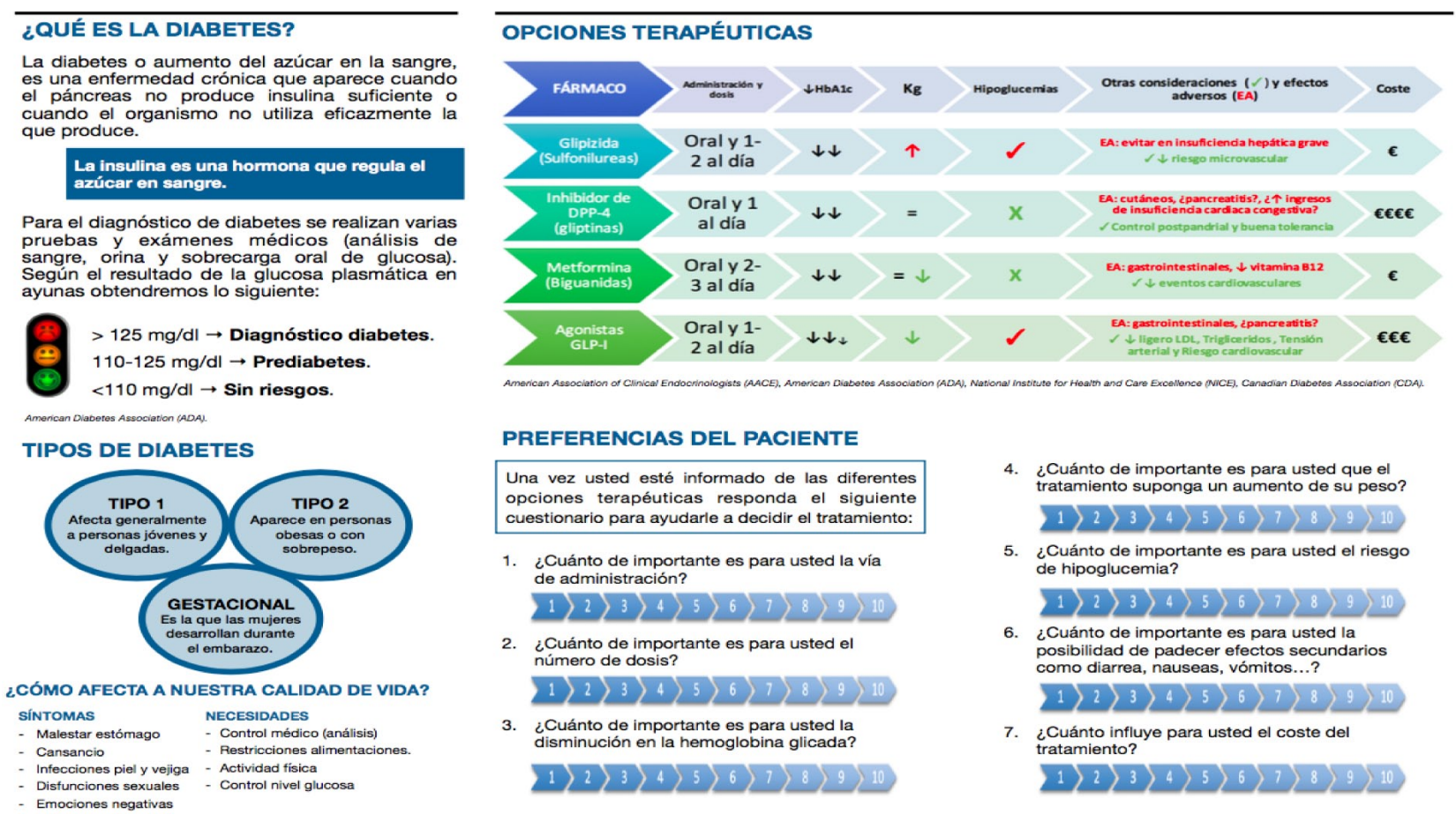

\section{Discusión y conclusiones}

Se han elaborado muchas herramientas a lo largo del tiempo para abordar y ofrecer información de la diabetes a los pacientes, pero no todas han resultado ser utiles. Por eso se ha considerado de vital importancia poner a prueba nuestro resultado. Primero se ha realizado una autoevaluación en la que se ha estudiado si esta herramienta es realmente un instrumento de autoayuda, supone una mejora de la corresponsabilidad médico-paciente a la hora de tomar decisiónes terapéuticas, y además fomenta el principio de autonomía, aumentando la confianza del paciente y sobre todo mejorando su adherencia al tratamiento.

Además, la herramienta ha sido validada según los criterios de evaluación y validación de las herramientas de ayuda para la toma de decisiones, basado en los criterios de The International Patient Decision Aid Standards (IPDAS) Collaboration Quality Dimensions: checklist y manual. El único criterio no cumplido ha sido el de conflicto de intereses. Sin embargo, no consta de este apartado dado que no es valorable que se presente un conflicto de interés en herramientas de este tipo.

Por último, los meses de abril y mayo se ha evaluado esta herramienta en los centros de atención primaria a aquellos pacientes con diabetes de tipo 2 que acudían a consulta con el objetivo de cambiar su tratamiento. El resultado ha sido muy positivo y favorable, ya que los pacientes han afirmado entender claramente la información proporcionada y aumentar su nivel de interés frente a su patología, al haberles dado un papel fundamental en la toma de decisiones frente a la misma.

Con todo esto, la finalidad del trabajo ha sido la elaboración de un instrumento de ayuda para que los pacientes conozcan más acerca de su patología y puedan tomar decisiones según sus preferencias, aumentando su confianza en el sistema de salud y la adherencia al tratamiento.

Concluyendo como frase final, «el médico proporciona la información, el paciente valora la opción y ambos toman la decisión». 


\section{Referencias bibliográficas}

American Association of Clinical Endocrinologists |. (s. f.). Recuperado el 8 de noviembre de 2017, a partir de https://www.aace.com/

American Diabetes Association®. (s. f.). Recuperado 8 de noviembre de 2017, a partir de http://www.diabetes.org/es/

Brunton, L., Parker, K., Blumenthal, D. y Buxton, I. (2009). Manual de farmacología y terapéutica. Goodman y Gilman: Mc Graw Hill.

Bosch J.M., (2012). La toma de decisiones conjuntas en medicina: una difícil asignatura. Aten Primaria: 44: 385-6.

Coulter A., Parsons S. y Askham J (2008). Where are the patients in decision-making about their own care? World Health Organization 2008 and World Health Organization, on behalf of the European Observatory on Health Systems and Policies.

Depablos, P., Salguero, E., Mata, J., Derivas, B., García, R. y Viguera, P. (2014). Quality of Life and Satisfaction with Treatment in Subjects with Type 2 Diabetes: Results in Spain of the Panorama Study. Endocrinología Y Nutrición: Órgano De La Sociedad Espanola De Endocrinologia Y Nutricion 61, no. 1: 18-26.

Drug and Therapeutics Bulletin. (2013). An introduction to patient decision aids. BMJ (Clinical Research Ed.), 347, f4147.

Flores, J., Armijo, J.A., y Mediavilla, A. (2008). Farmacología Humana. Masson-Salvat: $5^{\circ}$ Edición.

Jiménez De Gracia L., Ruiz R., Gavilán E., Hueso C., Cano Caballero D. y Alba Dios MA (2012). Opiniones de los médicos de familia acerca de la implicación de los pacientes en la toma de decisiones: un estudio con grupos focales. Aten Primaria. 44(7): 379-386.

Ministerio de sanidad, servicios sociales e igualdad. (s. f.). Manual con criterios de evaluación y validación de las Herramientas de Ayuda para la Toma de Decisiones (Basado en los criterios de The International Patient Decision Aid Standards (IPDAS) Collaboration Quality Dimensions: checklist y manual).

Centro de investigación biomédica en Red de Diabetes y Enfermedades Metabólicas asociadas. (s. f.). Prevalencia de la Diabetes en España. Estudio Di@bet.es.

Risk Factors. (s. f.). Recuperado el 8 de noviembre de 2017, a partir de http://www.diabetes. ca/about-diabetes/risk-factors

Ruiz, R., Peralta, L., Pérula, L.A., Olloqui, J., Carrión T., Sobrino, A., y cols. (2012). Opiniones y percepciones de los pacientes sobre su participación en la toma de decisiones en las consultas de medicina de familia. Aten Primaria. 44: 5-12.

Salgado, M., Franch, J., Pallas, M., Oriol, C., Grau, J. y Castellà, J. (s. f.). Estadísticas y causas de mortalidad en la diabetes tipo 2 | Atención Primaria. Recuperado el 8 de noviembre de 2017, a partir de http://www.elsevier.es/pt-revista-atencion-primaria-27-articuloestadisticas-causas-mortalidad-diabetes-tipo-S0212656701788750

Signs y Symptoms. (s. f.). Recuperado el 8 de noviembre de 2017, a partir de http://www.diabetes.ca/about-diabetes/signs-and-symptoms

Types of Diabetes. (s. f.). Recuperado 8 de noviembre de 2017, a partir de http://www.diabetes.ca/about-diabetes/types-of-diabetes 